\title{
A comparison of discrimination and identification of vibrotactile patterns
}

\author{
DAVID T. HORNER and JAMES C. CRAIG \\ Indiana University, Bloomington, Indiana
}

\begin{abstract}
Both discrimination and identification tasks have been used to assess subjects' abilities to perceive vibratory spatial patterns presented to the skin. The present study examined discrimination and identification performance under comparable conditions. In Experiment 1, subjects attempted to discriminate a pair of patterns on some blocks of trials and to identify both members of a pair on other blocks. For both tasks, the time between the members of the pair was varied. Discrimination performance could be predicted accurately from identification data. Analysis of performance on identification trials indicated that subjects used discriminability information to identify pairs. In Experiment 2, discrimination and identification were compared when the temporal separation between patterns was fixed and a masking stimulus followed each pattern after a variable delay. Results suggest that temporal masking, rather than the time available for processing pattern information, is the major limitation in both discrimination and identification of sequences of tactile patterns.
\end{abstract}

The skin rarely processes a single pattern isolated temporally from other patterns. The hands and fingers explore the surface of an object by moving across successive surface areas. Thus, sequences of tactile, spatial patterns are produced. Sometimes, a tactile surface is explored to locate the place where the surface changes, which may be viewed as a discrimination task: Are successive patterns the same or different? At other times, a surface is explored to reach a decision about what is being felt, an identification task. These two measures of performance-discrimination and identification-have been examined in a number of studies using vibratory spatial patterns (discrimination-Craig, 1985a, 1985b; Geldard \& Sherrick, 1965; Gilson, 1968; Gottheil, Cholewiak, \& Sherrick, 1978: identification-Bliss, Crane, Link, \& Townsend, 1966; Bliss \& Linvill, 1966; Craig, 1976, 1980, 1983a, 1983b, 1985a, 1985b; Craig \& Evans, 1987; Evans, 1987; Evans \& Craig, 1986).

Several studies have examined the effect of temporal separation of two patterns of a pair on the identification of either the first or second member of the pair. As temporal separation increases from 9 to $800 \mathrm{msec}$, pattern identification improves, an improvement that has been attributed to reduced temporal masking at longer temporal separations (Craig, 1983a, 1983b; Craig \& Evans, 1987; Evans \& Craig, 1986). The opposite effect, reduced performance with increasing temporal separation, might be

This research was supported by Grant NS- 09783 from the National Institutes of Health. The authors wish to thank Roger Rhodes for his assistance in conducting these experiments. The authors also wish to thank Martha Teghtsoonian and two reviewers for their comments on this manuscript. Correspondence concerning this article should be sent to David T. Horner or James C. Craig, Department of Psychology, Indiana University, Bloomington, IN 47405. expected in a discrimination task. Studies with visual and auditory stimuli have shown that discrimination performance declines as the temporal separation between members of a pair of stimuli is increased (Bindra, Williams, \& Wise, 1965; Krueger \& Shapiro, 1985; Mitchell, 1972; Smith \& Nielson, 1970). A common explanation for declining performance is that a representation of the first stimulus decays during the period before it is compared with the second stimulus, at least in the case of stimuli not verbally encoded (Krueger, 1978; Posner, Boies, Eichelman, \& Taylor, 1969).

Contrary to what might be expected, discrimination performance for vibratory patterns has been shown to improve as the temporal interval between patterns is increased from 9 to 300 msec (Cholewiak \& Craig, 1984; Craig, 1983a). This improvement is particularly surprising given the stimulus display conditions. For example, Craig (1983a) presented patterns to the subject's fingerpad using a 144-pin vibrotactile array and varied the time between the onsets of the two patterns (stimulus onset asynchrony, or SOA). A same-pattern trial at brief SOAs consisted of a continuous single pattern, a relatively unchanging stimulus, whereas a different-pattern trial contained two patterns that differed in the number and the spatial configuration of activated pins in the arrays. At brief SOAs performance was poor (less than $72 \%$ correct), even though the first pattern overlapped temporally with the second. At longer SOAs, temporal overlap no longer occurred, and both same-pattern and differentpattern trials contained two temporally distinct patterns. The improvement in discrimination performance with increasing SOA has been attributed to a decline in temporal masking (Cholewiak \& Craig, 1984; Craig 1983a).

Because discrimination was surprisingly poor at brief SOAs and identification was also poor at brief SOAs and 
improved with increasing SOA, Cholewiak and Craig (1984) and Craig (1983a) speculated that subjects must identify patterns in order to discriminate them. These two studies compared discrimination performance with identification performance in a masking task that involved naming only a single pattern. Craig (1983a) found that identification performance underpredicted discrimination performance. Cholewiak and Craig (1984) rank ordered discrimination and identification performance across several conditions. They found that relatively good performance on one task did not necessarily predict relatively good performance on the other.

The present study provided a direct, quantitative comparison of subjects' abilities to discriminate and to identify a pair of patterns and addressed the question of whether masking is a major factor that limits performance at brief SOAs. Experiment 1 compared discrimination and identification performance directly. Placing these two tasks in the broader context of processing of successive tactile patterns, an additional experiment compared discrimination and identification under similar conditions of masking and temporal separation.

\section{EXPERIMENT 1}

Pairs of letter patterns were presented to subjects, and, as in previous studies of discrimination and identification, performance was examined over a range of SOAs. The use of SOAs long enough to minimize masking, up to $6,400 \mathrm{msec}$, was expected to aid in comparing discrimination and identification. Specifically, we expected that discrimination performance might decline at longer SOAs because of a decaying representation of the first pattern, and that identification performance might not decline at longer SOAs because the first pattern is named.

\section{Method}

Subjects. The subjects were 4 students, 3 women and 1 man, who were employed in the laboratory. All had previous experience in several discrimination tasks and in a letter identification task.

Apparatus. The patterns, all 26 uppercase letters of the alphabet, were presented on a vibratory array. The array measured $1.1 \times$ $2.7 \mathrm{~cm}$ and consisted of 144 pins arranged in 24 rows and 6 columns. All letters contained pins within the upper (distal) 18 rows of the array and, with the exception of "I" and "J," all 6 columns of the array, an area approximately $1.1 \times 2.0 \mathrm{~cm}$. The array was interfaced with a DEC PDP-11/34 computer, which controlled the amplitude and duration of vibration of the individual pins. Each of the pins on the array vibrated at $230 \mathrm{~Hz}$. The intensity of the vibration was set at a comfortable level, $33 \mathrm{~V}$ to the driver circuits, for all subjects and conditions. Further details about the vibrotactile array may be found in a previous publication (Craig, 1980).

Procedure. The subject was seated, and rested the distal pad of the left index finger on the array. The subject wore earphones through which low-pass filtered noise was fed to mask auditory cues from the array. A trial began when the subject pressed a key on a keyboard located next to the array, and a cue stimulus consisting of the topmost left and topmost right pins in the array was activated for $13 \mathrm{msec}$. The cue stimulus also served to ensure that the finger was correctly positioned on the array. One second after the cue stimulus, the first letter was presented for $26 \mathrm{msec}$, which was followed after a temporal interval (SOA) by the second letter, also presented for $26 \mathrm{msec}$. The two letters were the same on half of the trials and different on half of the trials, at random. The subjects responded by selecting appropriate keys on the keyboard and received feedback on a CRT screen.

In the discrimination task, the subjects were told that on half of the trials a pair of identical letters would be presented (a letter-same trial) and on half of the trials a pair of different letters would be presented (a letter-different trial). The subjects responded by pressing " $S$ " or " $D$ " on the keyboard (a "same" response or a "different" response, respectively). Trial-by-trial feedback was provided: either the word "correct" appeared on the screen, or, when the response was incorrect, an " $S$ " or " $D$ " appeared, corresponding to the correct choice. The SOAs were $52,100,400,800,1,600$, 3,200 , and $6,400 \mathrm{msec}$. Seven blocks of 40 trials, each block testing a different SOA, were presented randomly within the experimental session.

The same testing procedure was used in the identification task, except that the subject had to identify both letters in the pair, rather than discriminate them. The subject was permitted to respond only after both letters were presented. Feedback was provided, with the word "correct" appearing only if the subject identified both letters in the correct order. Feedback for incorrect responses was a display of the correct pair of letters on the CRT screen, their leftto-right spatial order matching the temporal order in which they were presented. The same SOAs were tested as in the discrimination task. The discrimination and identification tasks were presented on alternate days. All seven SOAs were tested in each session.

\section{Results and Discussion}

The results from the discrimination task were used to calculate $P(C)$ max at each SOA. This statistic (McFadden, 1970), used in previous studies of tactile discrimination (Cholewiak \& Craig, 1984; Craig, 1983a), minimizes effects of response bias. The percentage of " $S$ " and " $D$ " responses varied less than $6 \%$ from that expected by chance, except at the $400-\mathrm{msec}$ SOA, where the percentage of " $D$ " responses was $38 \%$. The data were scored in terms of hits (e.g., responding " $D$ " on a letterdifferent trial) and false alarms (e.g., responding " $D$ ", on a letter-same trial). Hits and false alarms were used to calculate $d^{\prime}$ and $P(\mathrm{C}) \max$. Using $P(\mathrm{C})$ max changed the percentage of correct responses only slightly, by less than $2 \% . P(\mathrm{C}) \max$ and the percentages of correct responses on letter-same and letter-different trials are plotted as a function of SOA in Figure 1. Each point on the $P(C)$ max function represents a total of 1,120 trials from 4 subjects. Each point on the letter-same and letterdifferent functions represents, on average, 560 trials from 4 subjects. The standard errors for any plotted point were less than $3 \%$.

Figure 1 shows that the decline in $P(C) \max$ at SOAs beyond $800 \mathrm{msec}$ was caused by declining accuracy on letter-same trials. A decline in performance when the temporal interval between identical stimuli is increased has also been found using visual spatial patterns (Krueger \& Shapiro, 1985; Mitchell, 1972; Smith \& Nielson, 1970), and tones (Bindra et al., 1965). Krueger (1978) suggested that an increase in internal noise associated with a long interstimulus interval distorts the processes involved in encoding and storing representations of the patterns, thus making it more likely for a pair of identical stimuli to appear different than for a pair of different stimuli to ap- 


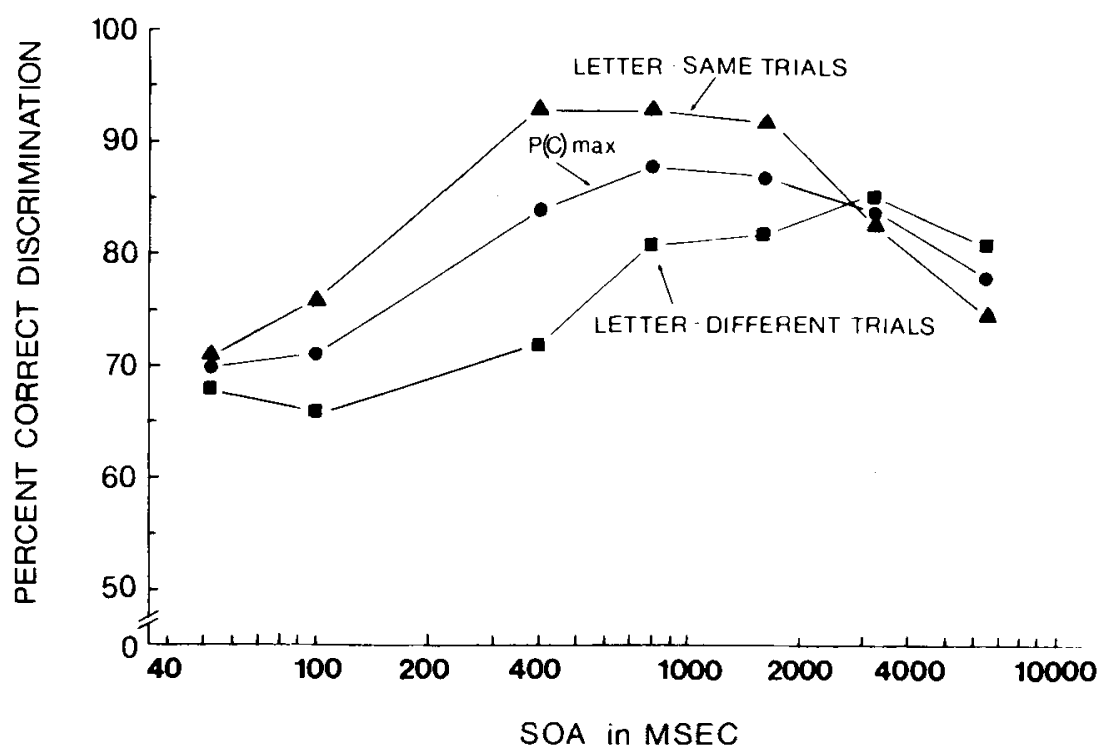

Figure 1. Percentage of correct responses, $P(C) \max$, in a discrimination task as a function of stimulus onset ansynchrony. Also shown are percentages of correct responses on letter-same and letter-different trials. Average data for 4 subjects.

pear identical. If the present decline in performance on letter-same trials was caused by loss of spatial information about the first pattern, then the two patterns should have felt different more often than they felt the same. Each subject showed an increased tendency to respond " $D$ " at longer SOAs. An analysis of variance performed on the data from the four longest SOAs showed that this in- crease in the proportion of " $D$ " responses, from an average of $44 \%$ at the $800-\mathrm{msec}$ SOA to an average of $55 \%$ at the 6,400 -msec SOA, was significant $[F(3,9)=11.51$, $p<.002]$.

Overall percentage of correct identification of both letters as a function of SOA is shown in the middle curve of Figure 2. Each point represents a total of 1,120 trials

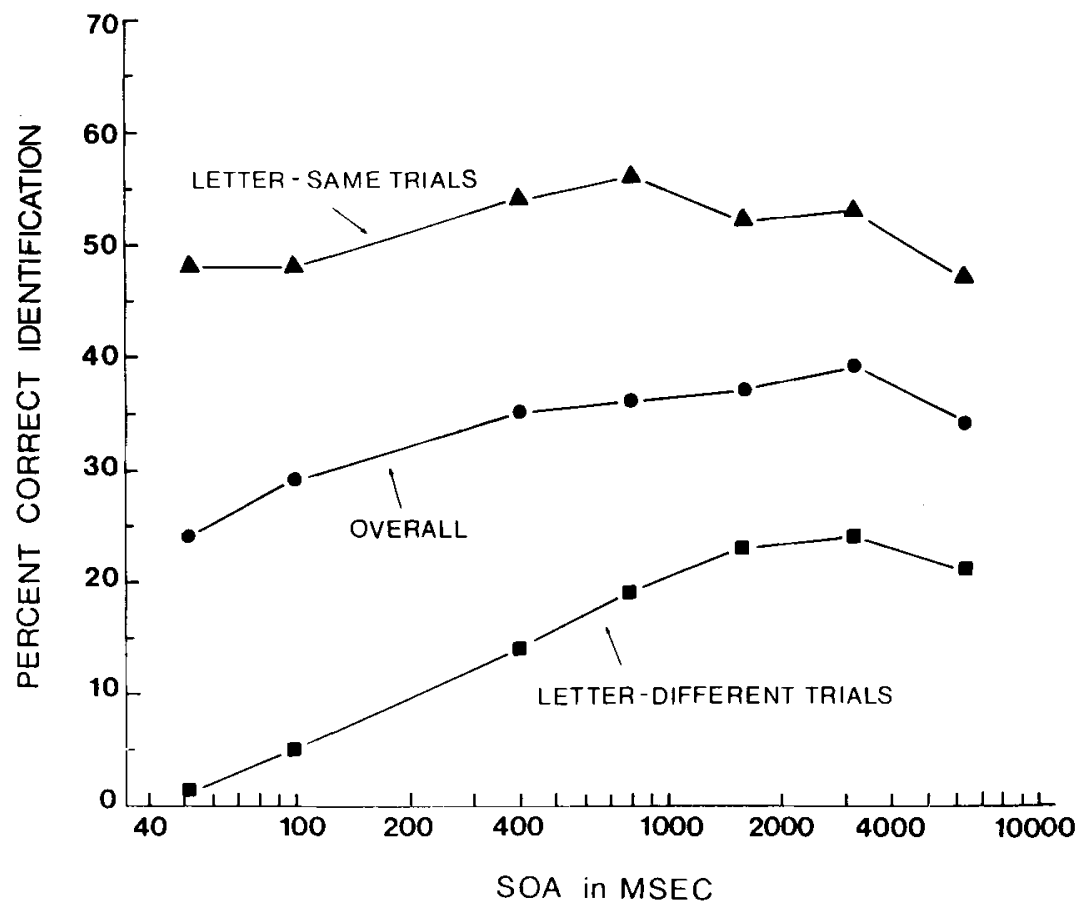

Figure 2. Percentage of correct responses in an identification task as a function of stimuls onset asynchrony. Also shown are percentages of correct responses on letter-same and letterdifferent trials. Average data for 4 subjects. 
from 4 subjects. Figure 2 also shows percent correct identification on letter-same and letter-different trials. Each point on these curves represents, on average, 560 trials from 4 subjects. The standard errors for any plotted point ranged from $1 \%$ to $4 \%$.

The shape of the overall identification function out to approximately $1 \mathrm{sec}$ is similar to the shape of a temporal masking function, in which performance in identifying a single pattern improves as the time between the pattern and masker increases. Performance in identifying a letter based on its temporal position in the pair was analyzed, and the results were consistent with previous temporal masking studies (Bliss et al., 1966; Craig, 1976, 1978, 1980, 1982, 1983a, 1983b; Craig \& Evans, 1987; Evans, 1987). Specifically, on letter-different trials, there were $4 \%$ to $7 \%$ more errors in identifying the first letter than the second at the two briefest SOAs, 52 and $100 \mathrm{msec}$, and $2 \%$ to $9 \%$ more errors in identifying the second letter than the first at longer SOAs. Temporal masking is also indicated by poor performance on letter-different pairs at brief SOAs. However, very little masking occurred on letter-same trials.

One reason for testing longer SOAs was to see whether the discrimination and identification functions would diverge because of possible differences in how the patterns are stored in memory in the two tasks. The results are not clear-cut on this issue. The discrimination and identification functions, the middle functions in Figures 1 and 2, both decline somewhat at longer SOAs. A separate repeated measures analysis of variance was performed for each task using data from the 800- and 6,400msec SOAs. Session-by-session percentage correct for each subject was used in the analysis. Discrimination performance declined significantly $[F(1,3)=17.52, p<$ $.02]$, and identification performance did not $[F(1,3)=$ $2.88, p>.19$ ]. However, considering the similar shape of the two functions, no strong conclusions can be made about differences in memory storage in the two tasks.

The main purpose of Experiment 1 was to compare the discriminability and identifiability of a pair of letters. To estimate how well subjects were "discriminating" in the identification task, the identification data were scored in the following way. If a subject responded with two identical letter names on a letter-same trial, or with two distinct names on a letter-different trial, the trial was scored as a correct discrimination response. These inferred discrimination scores were used to estimate $P(\mathrm{C}) \max$. The estimated and obtained discrimination $P(C)$ max functions are shown for each subject in Figure 3. Overall identification performance is also plotted for each subject. Discrimination functions are referred to the left-hand ordinates, and identification functions to the right-hand ordinates.

The estimated and obtained discrimination $P(C) \max$ functions (the two upper functions in each panel of Figure 3) overlap closely. Given that identification performance, scored in the manner described above, accurately predicts discrimination performance, one might be tempted to conclude that subjects identify each mem- ber of a pair of patterns in order to discriminate them. Such a conclusion seems unwarranted. With only the information in Figure 3, one might just as easily conclude the reverse: that subjects discriminate the two patterns to aid their identification of the pair.

Regardless of how the two processes might be related, identification performance provides sufficient information for predicting discrimination performance. The results shown in Figure 3 are neutral on the question of what strategies the subjects use: Subjects may be discriminating members of a pair on identification trials or making covert identification responses on discrimination trials. However, the fact that identification performance can predict discrimination performance suggests that instructing subjects either to discriminate or to identify does not alter their sensitivity in any quantitative way.

In arriving at the estimated discrimination functions in Figure 3, many incorrect identification responses were treated as correct discrimination responses. There were $67 \%$ incorrect letter pair identification responses and, of these incorrect identification responses, $69 \%$ were treated as correct discriminations. The data can be reanalyzed to treat only correct identification responses as correct discrimination responses and to consider incorrect identification responses as guesses, (i.e., assume $50 \%$ correct discrimination performance). The data analyzed in this way are presented in Figure 4, along with the obtained discrimination function from Figure 1, and show a consistent underestimation of discrimination performance. Hence, to hold the view that subjects identify patterns in order to discriminate them requires that subjects base their discrimination judgments on large numbers of trials in which the identification response is incorrect.

The opposite view, that subjects discriminate pairs of stimuli and use that information in their identification task, implies that subjects do not process each member of the pair independently. Analyzing the identification data according to letter-same and letter-different pairs indicates that, at least with regard to letter-same pairs, the stimuli are indeed not processed independently. The probability of correctly identifying a pair was estimated by taking the product of the probabilities of correctly identifying each member of the pair (the product rule estimate). This estimate was made at each SOA and is plotted in Figures 5 and 6 for letter-different and letter-same trials, respectively. Performance in correctly identifying each member of the pair and the pair itself is included in each figure. The functions labeled "letter pair" in these two figures are the letter-different and letter-same functions from Figure 2. The close fit between the estimated and obtained letter pair functions in Figure 5 agrees with the assumption of independence when two different stimuli are presented. However, Figure 6 shows that performance on pairs of identical letters is clearly not estimated accurately by assuming independent identification judgments. Nonindependence on letter-same trials implies that subjects are aware that the two patterns are identical, or, in other words, that subjects are discriminating the patterns. 

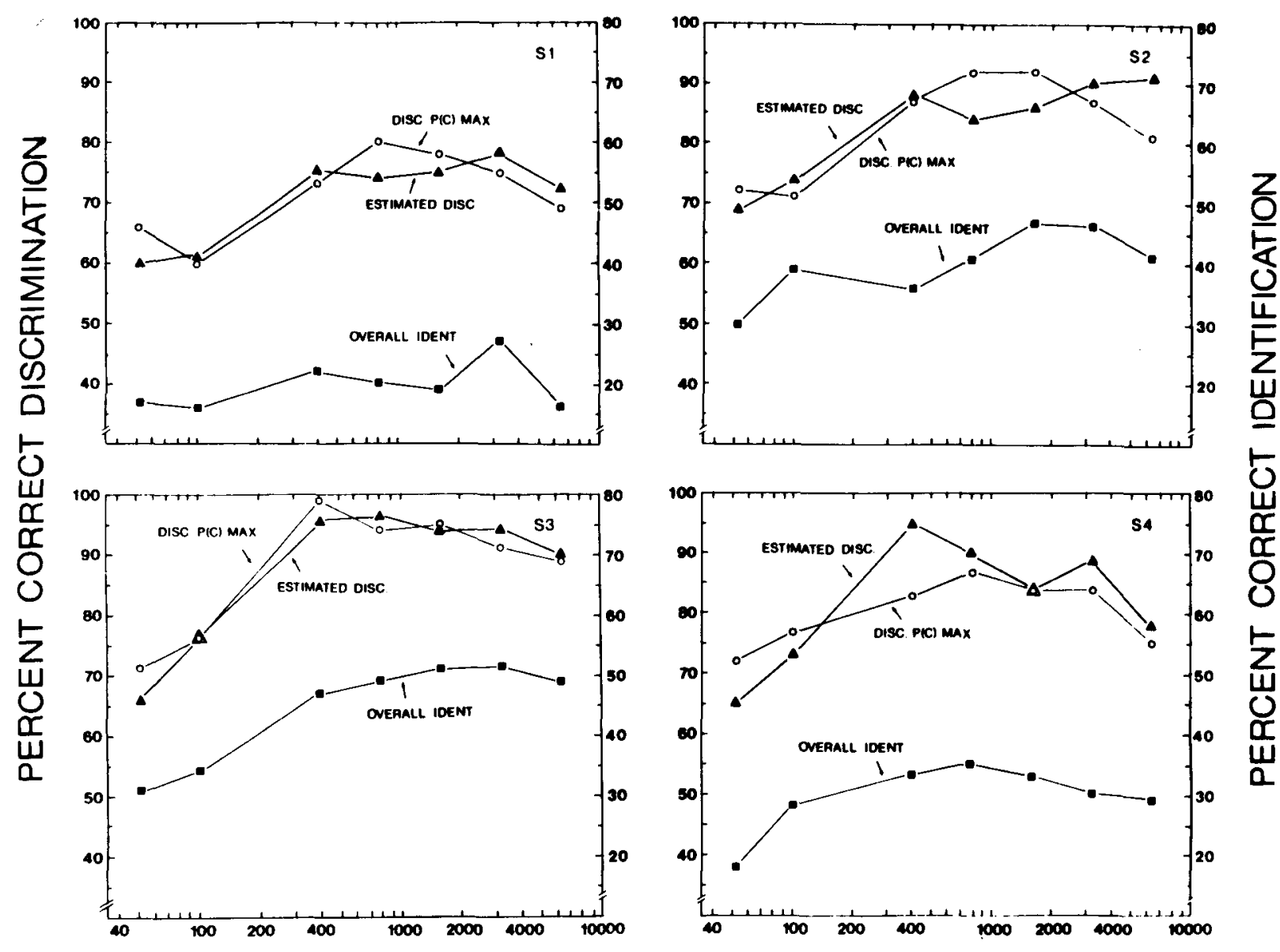

\section{SOA in MSEC}

Figure 3. Results from Experiment 1 showing individual subjects' discrimination $P(C)$ max (referred to left vertical axis in each panel) and overall identification responses (referred to right axis in each panel) as a function of SOA. Also shown are the percentages of correct discrimination responses estimated from the identification data (referred to left axis in each panel).

The results of an additional experiment support the idea that information about discriminability is used to identify letter pairs. The identification task of Experiment 1 was repeated (referred to as the no-message condition), and a second identification condition (the message condition) was added. In the message condition, before and during each trial, a message was presented on the CRT screen in front of the subject indicating whether the two letters were the same or different. The message and no-message conditions were presented on alternate days. Each condition tested SOAs of $52,100,400,800,3,200$, and $12,800 \mathrm{msec}$. Four subjects were tested, each receiving 280 trials per SOA in each condition. An analysis of variance was performed on the data. Performance in the message condition was significantly higher than in the nomessage condition, but only on letter-same trials $[F(1,3)=34.56, p<.01]$; that is, knowledge of the relationship between the two letters aided identification only when the two letters were identical. If subjects could perfectly identify the first member of a pair and knew that the second member was identical to the first, their identification of the pair would be perfect. However, correctly identifying the first letter and knowing that the second was different would provide little help to the subjects because the number of possible alternatives for different letters was large.

\section{EXPERIMENT 2}

The results of Experiment 1 suggest that masking might have been responsible for subjects' difficulty in responding to pairs of letters when the temporal separation was brief. However, subjects' difficulty might also have been due in part to insufficient time to process the pair of patterns. The rate at which tactile patterns were presented may have exceeded the skin's channel capacity.

In Experiment 2, the time between the pair of letters was held constant at $1,200 \mathrm{msec}$. Therefore, the number of letters identified or discriminated per unit time-a measure of channel capacity-was held constant. What was 


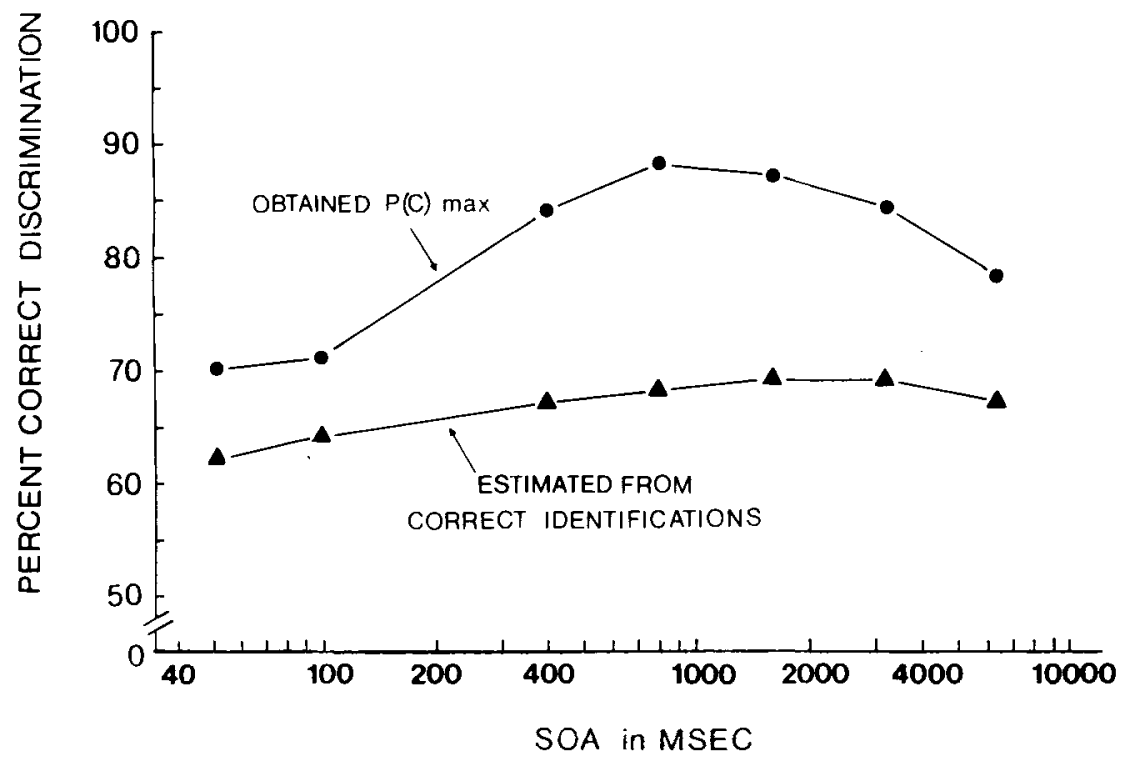

Figure 4. Percentage of correct discrimination responses, $P(C) \max$, and the percentage of correct discrimination responses estimated from correct identification (see explanation in text).

varied was the time between each letter and a backward masking stimulus. Figure 7 illustrates the experimental paradigm. If channel capacity is the only limit on processing of sequential patterns, then performance should remain unchanged as a function of the masker delay. Because previous work has so strongly implicated temporal masking as a limiting factor, performance is likely to change, but the degree of change may be used to gauge the importance of masking in Experiment 1. With the two letters presented $1,200 \mathrm{msec}$ apart, channel capacity should not limit performance, and the prediction is that, as the interval between letter and masker is increased, less

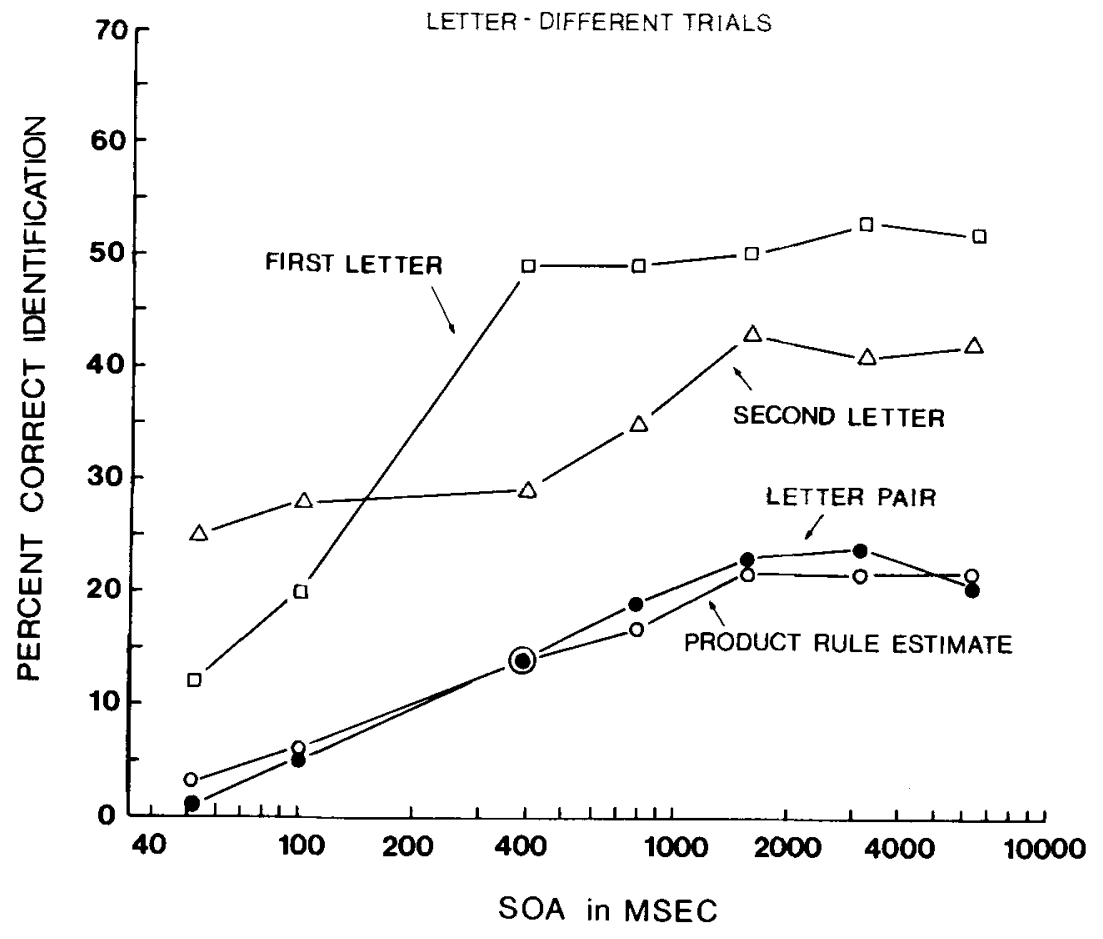

Figure 5. Obtained and estimated percentages of correct identification of a pair of letters on letter-different trials as a function of SOA. Also shown are percentages of correct identification of each member of a pair. 


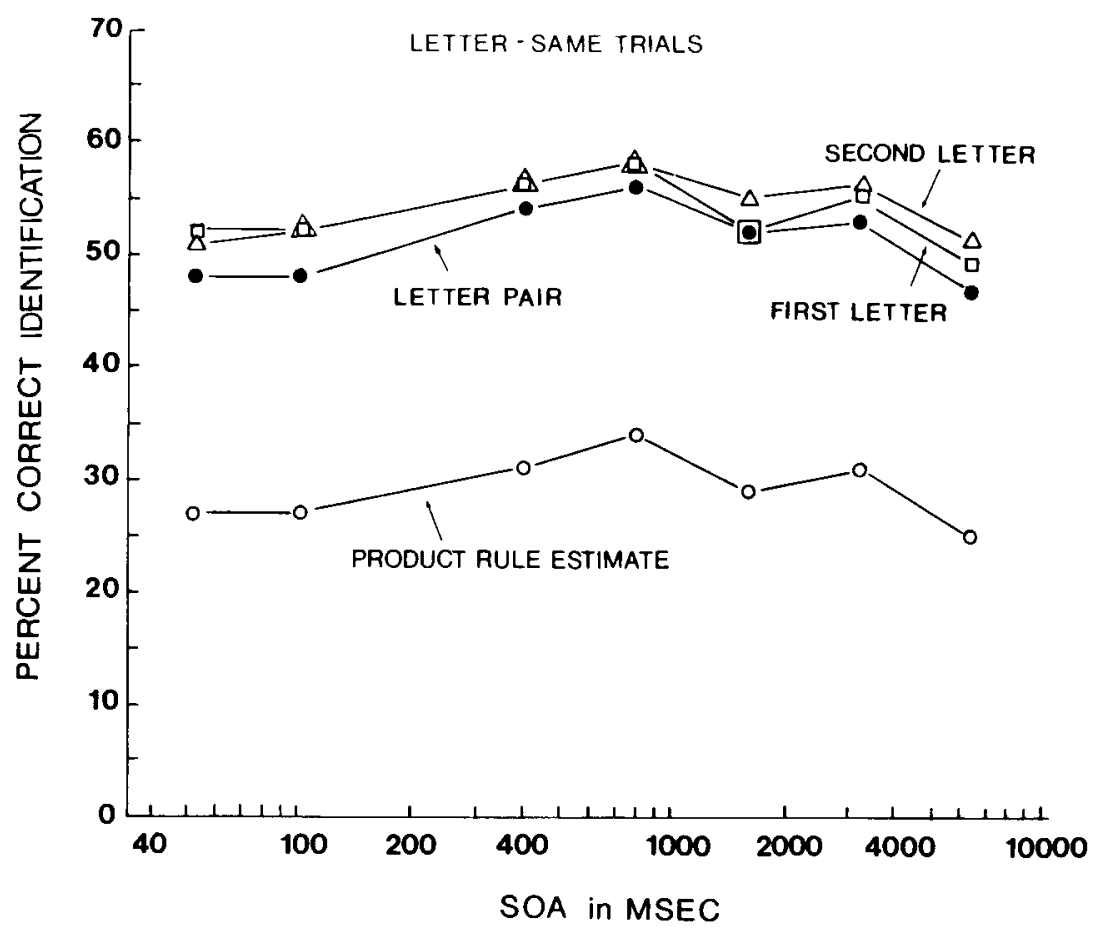

Figure 6. Obtained and estimated percentages of correct identification of a pair of letters on letter-same trials as a function of SOA. Also shown are percentages of correct identification of each member of a pair.

improvement in performance would be observed than in Experiment 1. There should be some improvement because of a decline in masking, but it would not be augmented by increased processing time for the pair of letters.

The fixed temporal separation $(1,200 \mathrm{msec})$ between letters was selected on the basis of a previous experiment with letter stimuli (Craig \& Evans, 1987), which showed that very little masking occurred when masker and target were separated by $1,200 \mathrm{msec}$, even when the masker was more intense than the target. Therefore, very little interference should be caused by interactions between the two letters. A relatively long temporal separation also should allow subjects to perceive the first letter before arrival of the second. Previous results in our laboratory showed that subjects in discrimination and identification tasks usually respond in less than $1 \mathrm{sec}$.

\section{Method}

Subjects. Three women and 1 man served as subjects and were selected as in Experiment 1. One of the subjects had participated in Experiment 1.

Apparatus. The same apparatus was used as in Experiment 1.

Procedure. The same stimuli and general testing procedures used in Experiment 1 were used in the discrimination and identification conditions, with the following modifications: One second after the cue stimulus, the first letter was presented. An energy masker, in which all the pins in the upper 18 rows of the array were activated simultaneously, was presented at a variable delay following the onset of the first letter. The second letter was always presented $1,200 \mathrm{msec}$ after the onset of the first letter. An energy masker followed the onset of the second letter at the same delay used for the first masker.

Energy maskers, rather than pattern maskers, were used to maintain a constant masking stimulus and to avoid forward masking of the second letter. Pattern maskers, which are composed of parts of letters, interfere with target identification over longer temporal separations than do energy maskers (Craig \& Evans, 1987). Preliminary results showed that identification performance was at chance levels for the briefest delays. Therefore, the duration of the letter stimuli was increased from 26 to $52 \mathrm{msec}$. The two letters were the same on half of the trials and different on half of the trials, at random. The subjects were instructed to ignore the energy maskers.

Two conditions were tested: discrimination, in which the subject pressed " $S$ " or " $D$ " on the keyboard, and identification, in which the subject named the two letters. Trial-by-trial feedback was provided. Blocks of $\mathbf{4 0}$ trials, each block testing a different masker delay, were presented randomly within the experimental session. The delays were $52,78,104,152,252$, and $352 \mathrm{msec}$. For an additional block of trials, no energy maskers were presented (nomasker trials). Each experimental session tested either discrimination or identification, and the conditions were tested on alternate days.

\section{Results and Discussion}

The same procedures used in Experiment 1 were used for calculating $P(\mathrm{C}) \max$ in the discrimination condition. $P(\mathrm{C})$ max is plotted as a function of the masker delay in the top curve of Figure 8. Each point represents a total

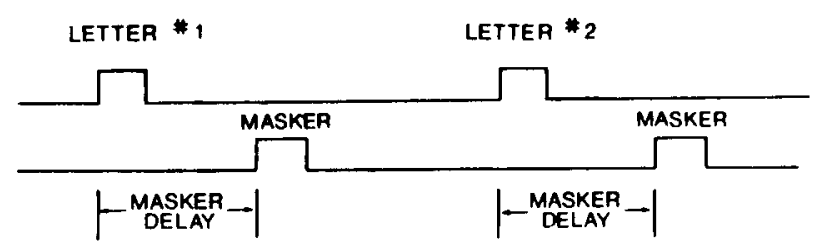

Figure 7. Illustration of the paradigm used in Experiment 2. 
of 960 trials from 4 subjects. Percentage of correct identification of both letters as a function of the masker delay is represented in the bottom curve of Figure 8. Each point represents a total of 960 trials from the 4 subjects. Discrimination and identification performance for the nomasker trials are also included in Figure 8. The standard errors of the means for percentage of correct identification and discrimination responses were less than $3 \%$. For comparison, identification performance and discrimination performance at the $1,600-\mathrm{msec}$ SOA of Experiment 1 are also plotted in Figure 8. Performance in Experiments 1 and 2 is comparable under similar conditions. Using the two methods described in Experiment 1, discrimination performance was estimated from identification performance and the same pattern of results was found; that is, if both correct and incorrect identification responses are used, the discrimination data are well estimated, but if only correct identification responses are used, the discrimination function is underestimated.

As expected, performance improved as the masker delay was increased, suggesting that channel capacity is not the sole factor limiting the ability to discriminate or identify a pair of patterns separated by a brief temporal interval. Did channel capacity have any significant role in limiting performance in Experiment 1 ? If it did, then performance in Experiment 2 should improve less with increasing temporal separation than in Experiment 1. However, the results were in the opposite direction. Discrimination performance increased 14 percentage points between the SOAs of 52 and $400 \mathrm{msec}$ in Experiment 1 (see Figure 1) compared with an increment of 17 percentage points between the masker delays of 52 and $350 \mathrm{msec}$ in Experiment 2. Identification performance improved 11 percentage points between the SOAs of 52 and $400 \mathrm{msec}$ in Experiment 1 (see Figure 2) compared with an increment of 22 percentage points between the masker delays of 52 and $350 \mathrm{msec}$ in Experiment 2. These results suggest that channel capacity was not overloaded in Experiment 1 . Thus, according to the present results, masking appears to be the major, if not the only, factor limiting performance at brief SOAs. This conclusion is consistent with previous results (Craig, 1985a). If masking is reduced by presenting a pair of patterns to fingers on opposite hands, performance does not decline at brief SOAs.

\section{GENERAL DISCUSSION}

The emphasis of this study was on the relationship between discrimination and identification of vibrotactile spatial patterns. The results are consistent in showing that subjects can discriminate a pair of patterns under conditions in which they cannot correctly identify the pair. Even when subjects incorrectly identify patterns, information about the discriminability of the pair is apparently available. These results are also consistent with visual matching studies that have concluded that identification requires a more detailed analysis of stimuli, involving memory and response factors, than does discrimination (Posner \& Mitchell, 1967; Proctor, 1981; Robinson, Brown, \& Hayes, 1964).

Craig (1983a) suggested that factors such as apparent motion (Sherrick, 1968a, 1968b; Sherrick \& Rogers, 1966) and saltation (Geldard, 1975; Geldard \& Sherrick, 1972) might provide cues as to whether two patterns occupy the same locations, and thus raise discrimination performance above that predicted from correct identification responses. Subjects report that such cues are available when vibrotactile spatial patterns are presented to the thigh

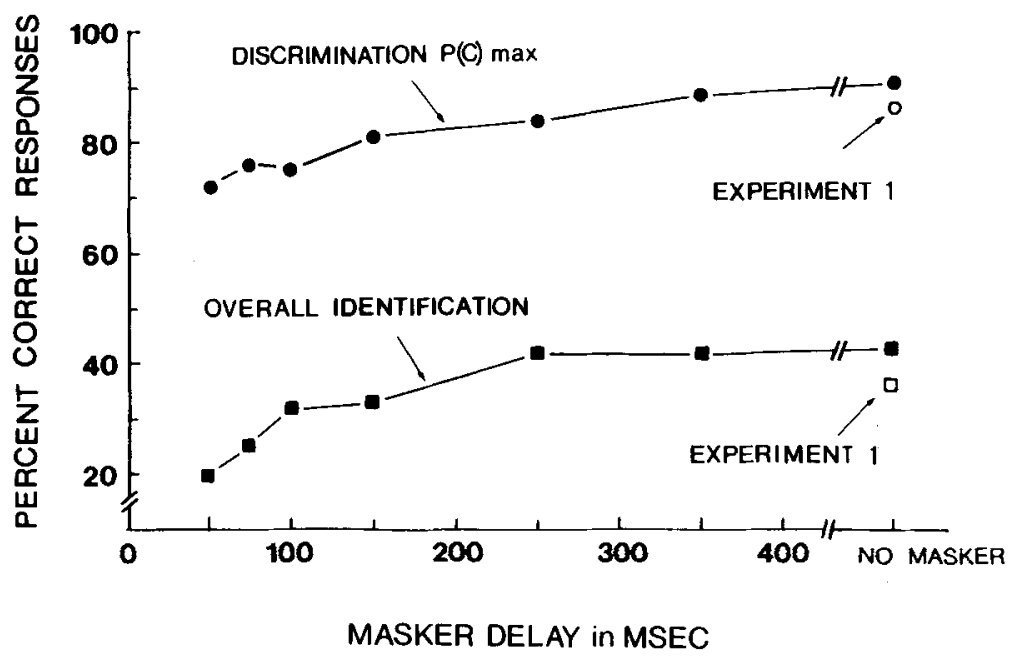

Figure 8. Percentage of correct discrimination responses, $P(C) \max$, and percentage of correct overall identification responses as a function of the delay between letter and masker. Discrimination $P(C) m a x$ and overall identification performance from the 1,600msec SOA of Experiment 1 are also shown. 
(Cholewiak, 1984). Presumably, on a letter-different trial, apparent motion and perhaps saltation between different features contained within each of the two patterns provide cues to aid discrimination. However, they operate only at relatively brief SOAs, and the present finding that discrimination performance is higher than predicted from correct identification, even at very long SOAs, suggests that they contribute little to discrimination.

What do the results of Experiments 1 and 2 tell us about the perception of sequences of vibrotactile patterns? The fact that subjects' sensitivity did not vary with instructions to identify or to discriminate suggests that improving the discriminability of patterns in a temporal sequence improves the subject's ability to name each pattern. However, as Bliss and Linvill (1966) pointed out, care should be taken in extrapolating results about perception of pattern pairs to the perception of longer sequences of patterns. First, the effects of long-term practice may alter the shapes of the discrimination and identification functions. Subjects in the present experiment received limited training with pattern pairs, whereas readers using the Optacon (Bliss, Katcher, Rogers, \& Shepard, 1970) and braille readers have much more experience with temporal strings of vibrotactile patterns. Second, perceiving long sequences of patterns may involve additional factors beyond those necessary for perception of pairs; for example, context cues should aid perception during reading, whereas in other tasks memory limitations may interfere with the perception of long sequences.

One motivation for the present study was the fact that discrimination performance is surprisingly poor under conditions that might logically be expected to improve discriminability. As noted in the introduction, one might expect the difference between a letter-same trial and a letterdifferent trial to be most obvious when the temporal separation between two patterns is brief, because the two patterns overlap temporally. Despite this expectation, the results of Experiment 1 suggest that, if such an advantage did exist, it was obscured by masking. Both discrimination performance and identification performance were poor at brief SOAs. The results of Experiment 2 indicated the importance of temporal masking in limiting performance under conditions in which at least one other source of possible interference-processing time-was ruled out. Taken together, the results of Experiments 1 and 2 support the view that temporal masking is a major factor responsible for poor discrimination and poor identification at brief SOAs.

The present study has implications for the evaluation of cutaneous communication systems, such as braille, Tadoma (Reed, Durlach, Braida, \& Schultz, 1982), and the Optacon. The fact that overall discrimination and identification performance varied similarly with changes in SOA suggests that similar processing may be involved in the two tasks. Similar processing is also suggested by previous results that have shown performance in both tasks to improve when either pattern duration is increased
(Cholewiak \& Craig, 1984) or pattern complexity is decreased (Craig, 1985a). Taken together, these results indicate that if a particular communication system leads to good discrimination of tactile spatial patterns, it may also lead to good identification of such patterns. However, the fact that Cholewiak and Craig (1984) found an imperfect match between discrimination and identification performance when rank ordered across several conditions argues for some caution in assuming that improved discriminability automatically leads to improved identifiability.

Testing subjects' abilities to discriminate using a particular communication system may serve as an efficient predictor of whether the system will be useful in identifying patterns. A discrimination task would be an efficient measure to use because, presumably, discrimination requires less training than identification. The extent to which this assumption is true might be revealed by examining performance changes in both tasks over time as subjects are trained on a set of tactile patterns.

\section{REFERENCES}

BindRA, D., Willuams, J. A., \& Wise, J. S. (1965). Judgments of sameness and difference: Experiments on decision time. Science, 150, 1625-1627.

Bliss, J. C., CRane, H. D., Link, S. W., \& Townsend, J. T. (1966). Tactile perception of sequentially presented spatial patterns. Perception \& Psychophysics, 1, 125-130.

Bliss, J. C., Katcher, M. H., Rogers, C. H., \& Shepard, R. P. (1970). Optical-to-tactile image conversion for the blind. IEEE Transactions on Man-Machine Systems, MMS-11, 58-64.

Buiss, J. C., \& LiNvill, J. G. (1966). A direct translation reading aid: Reading alphabetic shapes tactually. In R. Dufton (Ed.), Proceedings of the International Conference on Sensory Devices for the Blind (pp. 389-407), London, England: Arrowsmith.

Cholewiak, R. W. (1984, November). Cues in vibrotactile pattern discrimination. Paper presented at the 25th Annual Meeting of the Psychonomic Society, San Antonio, TX.

Cholewiak, R. W., \& Craig, J. C. (1984). Vibrotactile pattern recognition and discrimination at several body sites. Perception \& Psychophysics, 35, 503-514.

CraIG, J. C. (1976). Vibrotactile letter recognition: The effect of a masking stimulus. Perception \& Psychophysics, 20, 317-326.

CraIG, J. C. (1978). Vibrotactile pattem recognition and masking. In G. Gordon (Ed.), Active touch: The mechanism of recognition of objects by manipulation: A multi-disciplinary approach (pp. 229-242). Oxford, England: Permagon Press.

Craig, J. C. (1980). Modes of vibrotactile pattern perception. Journal of Experimental Psychology: Human Perception \& Performance, 6 , 151-166.

Craig, J. C. (1982). Vibrotactile masking: A comparison of energy and pattern maskers. Perception \& Psychophysics, 31, 523-529.

Craig, J. C. (1983a). The role of onset in the perception of sequentially presented vibrotactile patterns. Perception \& Psychophysics, 34, 421-432.

Craig, J. C. (1983b). Some factors affecting tactile pattern recognition. International Journal of Neuroscience, 19, 47-58.

Craig, J. C. (1985a). Attending to two fingers: Two hands are better than one. Perception \& Psychophysics, 38, 496-511.

CraIG, J. C. (1985b). Tactile pattern perception and its perturbations. Journal of the Acoustical Society of America, 77, 238-246.

Craig, J. C., \& Evans, P. M. (1987). Vibrotactile masking and the persistence of tactual features. Perception \& Psychophysics, 42, 309-317. 
Evans, P. M. (1987). Vibrotactile masking: Temporal integration, persistence, and strengths of representations. Perception \& Psychophysics, 42, 515-525.

Evans, P. M., \& CraIG, J. C. (1986). Temporal integration and vibrotactile backward masking. Journal of Experimental Psychology: Human Perception \& Performance, 12, 160-168.

GELDARD, F. A. (1975). Sensory saltation: Metastability in the perceptual world. Hillsdale, NJ: Erlbaum.

GelDard, F. A., \& Sherrick, C. E. (1965). Multiple cutaneous stimulation: The discrimination of vibratory patterns. Journal of the Acoustical Society of America, 37, 797-801.

Geidard, F. A., Sherrick, C. E. (1972). The cutaneous "rabbit": A perceptual illusion. Science, 178, 178-179.

Gilson, R. D. (1968). Some factors affecting the spatial discrimination of vibrotactile patterns. Perception \& Psychophysics, 3, 131-136.

Gottheil, E. F., Cholewiak, R. W., \& Sherrick, C. E. (1978). The discrimination of vibratory patterns on a tactile matrix. Bulletin of the Psychonomic Society, 11, 21-24.

Krueger, L. E. (1978). A theory of perceptual matching. Psychological Review, 85, 278-304.

Krueger, L. E., Shaptro, R. G. (1985). Effect of interstimulus interval and heterogeneity of difference on same-different judgments of visual patterns. Bulletin of the Psychonomic Society, 23, 43-46.

MCFADDEN, D. (1970). Three computational versions of proportion correct for use in forced-choice experiments. Perception \& Psychophysics, 8, 336-342.

MitChell, D. C. (1972). Short-term visual memory and pattern masking. Quarterly Journal of Experimental Psychology, 24, 394-405.
Posner, M. I., Boies, S. J., Eichelman, W. H., \& Taylor, R. L. (1969). Retention of visual and name codes of single letters. Journal of Experimental Psychology Monograph, 79(1, Pt. 2), 1-16.

Posner, M. I., \& Mitchell, R. F. (1967). Chronometric analysis of classification. Psychological Review, 74, 392-409.

Proctor, R. W. (1981). A unified theory for matching-task phenomena. Psychological Review, 88, 291-326.

Reed, C. M., Durlach, N. J., Braida., L. D., \& Schultz, M. C. (1982). Analytic study of the Tadoma method: Identification of consonants and vowels by an experienced Tadoma user. Journal of Speech \& Hearing Research, 25, 108-116.

Robinson, J. S., Brown, L. T., \& HAYES, W. H. (1964). Test of effects of past experience on perception. Perceptual \& Motor Skills, 18, 953-956.

Sherrick, C. E. (1968a). Bilateral apparent haptic movement. Perception \& Psychophysics, 4, 159-160.

ShERRICK, C. E. (1968b). Studies of apparent tactual movement. In D. R. Kenshalo (Ed.), The Skin Senses (pp. 331-344). Springfield, IL: Thomas.

Sherrick, C. E., \& Rogers, R. (1966). Apparent haptic movement. Perception \& Psychophysics, 1, 175-180.

SMITH, E. E., \& NiELSON, G. (1970). Representations and retrieval processes in short-term memory: Recognition and recall of faces. Journal of Experimental Psychology, 85, 397-405.

(Manuscript received September 21, 1987; revision accepted for publication July 13, 1988.) 